\title{
Fast Analytic Sampling Approximation from Cauchy Kernel
}

\author{
Youfa Li, ${ }^{1}$ Jing Shang, ${ }^{1}$ Honglei Yang, ${ }^{1}$ Gengrong Zhang, ${ }^{1}$ and Shouzhi Yang ${ }^{2}$ \\ ${ }^{1}$ College of Mathematics and Information Science, Guangxi University, China \\ ${ }^{2}$ Department of Mathematics, Shantou University, China
}

Correspondence should be addressed to Gengrong Zhang; 57018915@qq.com

Received 4 November 2015; Accepted 22 February 2016

Academic Editor: Kehe Zhu

Copyright (C) 2016 Youfa Li et al. This is an open access article distributed under the Creative Commons Attribution License, which permits unrestricted use, distribution, and reproduction in any medium, provided the original work is properly cited.

The paper aims at establishing a fast numerical algorithm for $\mathfrak{B}_{k}(f)$, where $f$ is any function in the Hardy space $H^{2}\left(\mathbb{T}^{d}\right)$ and $k$ is the scale level. Here, $\mathfrak{B}_{k}(f)$ is an approximation to $f$ we recently constructed by applying the multiscale transform to the Cauchy kernel. We establish the matrix expression of $\mathfrak{B}_{k}(f)$ and find that it has the structure of a multilevel Hankel matrix. Based on the structure, a fast numerical algorithm is established to compute $\mathfrak{B}_{k}(f)$. The computational complexity is given. A numerical experiment is carried out to check the efficiency of our algorithm.

\section{Introduction}

Approximation to a function (a time-continuous signal) by its samples is the heart of modern applied mathematics and engineering and has attracted much attention. Readers are referred to [1-5] for just a few references. For any function $f \in L^{2}\left(\mathbb{T}^{d}\right)$ with $\mathbb{T}=[0,2 \pi)$, it can be reconstructed by its Fourier coefficients as follows:

$$
f=\sum_{\mathbf{k} \in \mathbb{Z}^{d}} \hat{f}(\mathbf{k}) e^{i \mathbf{k} \cdot} .
$$

Here, $L^{2}\left(\mathbb{T}^{d}\right)$ is the space of $2 \pi \mathbb{Z}^{d}$-periodic and square integrable functions, equipped with the inner product

$$
\langle f, g\rangle=\frac{1}{(2 \pi)^{d}} \int_{\mathbb{T}^{d}} f\left(e^{i t}\right) \overline{g\left(e^{i t}\right)} d t,
$$

$$
\forall f, g \in L^{2}\left(\mathbb{T}^{d}\right),
$$

where $t:=\left(t_{0}, \ldots, t_{d-1}\right) \in \mathbb{T}^{d}, e^{i t}:=\left(e^{i t_{0}}, \ldots, e^{i t_{d-1}}\right)$, and $d t:=$ $d t_{0} \cdots d t_{d-1}$. Truncating the series in (1) leads to the classical Fourier sampling approximation. Specifically, for sufficiently large $N, f$ can be approximated by its Fourier coefficients $\left\{\hat{f}(\mathbf{k}):\|\mathbf{k}\|_{\ell_{2}} \leq N\right\}$ as follows:

$$
f \approx \sum_{\|\mathbf{k}\|_{e_{2}} \leq N} \hat{f}(\mathbf{k}) e^{i \mathbf{k}}
$$

where $\|\cdot\|_{\ell_{2}}$ is the Euclid norm of a vector in $\mathbb{R}^{d}$. From the point of view of feature characterization, (3) can be used to characterize some features such as instantaneous phase and frequency by those of the linear Fourier atoms $\left\{e^{i \mathbf{k} \cdot}: \mathbf{k} \in \mathbb{Z}^{d}\right\}$.

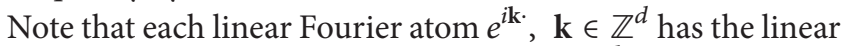
phase and constant frequency. Therefore, $e^{i \mathbf{k} \cdot}$ is time-stable. Then, the characterization from (3) implies that, whether $f$ is time-stable or not, its features are characterized by those of the linear Fourier atoms. However, when $f$ is not time-stable, the effectiveness of characterization by the linear atoms in (3) is inferior to that by nonlinear Fourier atoms [6-8].

Recently, an interesting sampling approximation from Takenaka-Malmquist (T-M) system, a special class of nonlinear Fourier atoms, has attracted much attention in the literature; for example, see $[9,10]$ and the references therein. Note that the T-M system is substantially generated from the Schmidt orthogonalization of Cauchy kernel $1 /(1-z)$, where $z \in \mathbb{D}:=\{z \in \mathbb{C},|z|<1\}$. In this sense, we say that the sampling approximation from the T-M system is substantially from the Cauchy kernel. Implementing the multiscale transform on the tensor product of the Cauchy kernel, Li and Qian [11] constructed the analytic sampling approximation to any function in the Hardy space $H^{2}\left(\mathbb{T}^{d}\right)$, where $H^{2}\left(\mathbb{T}^{d}\right)$, a subspace of $L^{2}\left(\mathbb{T}^{d}\right)$, is defined by

$$
H^{2}\left(\mathbb{T}^{d}\right):=\left\{f \in L^{2}\left(\mathbb{T}^{d}\right): \hat{f}(\mathbf{n})=0, \forall \mathbf{n} \in \mathbb{Z}^{d} \backslash \mathbb{N}_{0}^{d}\right\}
$$


with $\mathbb{N}_{0}$ being the set of nonnegative integers. Then, using the partial Hilbert transform, [11] generalized the approximation to $L^{2}\left(\mathbb{T}^{d}\right)$. A subsequent unsolved problem of the approximation in [11] is that the fast numerical algorithm has not been established. The aim of this present paper is to solve the problem by the fast computation theory of multilevel circulant matrices.

\section{Fast Algorithm for Multiscale Analytic Sampling Approximation}

2.1. Multiscale Analytic Sampling Approximation from Cauchy Kernel. Before introducing the approximation result in [11], we give the definition of the multiscale transform on $H^{2}\left(\mathbb{T}^{d}\right)$. For any $k \in \mathbb{N}_{0}$, define the $k$-scale transform $T_{k}^{\mathbf{m}}: H^{2}\left(\mathbb{T}^{d}\right) \rightarrow$ $H^{2}\left(\mathbb{T}^{d}\right)$ associated with the shift parameter $\mathbf{m} \in \mathbb{Z}^{d}$ by

$$
T_{k}^{\mathbf{m}} f=f\left(e^{i\left(\cdot-2 \pi 2^{-k} \mathbf{m}\right)}\right), \quad \forall f \in H^{2}\left(\mathbb{T}^{d}\right) .
$$

As for the parameter $\mathbf{m}$, we just need to focus on the case of m $\in \mathscr{L}_{k}$ due to the periodicity of $f$, where

$$
\begin{aligned}
\mathscr{L}_{k} & :=\left\{\left(m_{0}, \ldots, m_{d-1}\right): m_{v} \in\left\{0,1, \ldots, 2^{k}-1\right\}, v\right. \\
& \in\{0,1, \ldots, d-1\}\} .
\end{aligned}
$$

Suppose that $\left\{\phi_{k}\right\}_{k=0}^{\infty}$ is a sequence of $2 I_{d}$-nonstationary refinement functions in $H^{2}\left(\mathbb{T}^{d}\right)$; namely, they satisfy

$$
\phi_{k}=\sum_{\mathbf{m} \in \mathscr{L}_{k}} P_{k}(\mathbf{m}) T_{k}^{\mathbf{m}} \phi_{0}, \quad \forall k \in \mathbb{N}_{0}
$$

where $\left\{P_{k}(\mathbf{m})\right\}_{\mathbf{m} \in \mathscr{L}_{k}}$ belongs to $S\left(2^{k}\right)$, the space of $2^{k} \mathbb{Z}^{d}$ periodic complex-valued sequences. From the perspective of the Fourier coefficient, it is easy to check that (7) is equivalent to

$$
\widehat{\phi_{k}}(\mathbf{n})=\widehat{P_{k}}(\mathbf{n}) \widehat{\phi_{0}}(\mathbf{n}), \quad \forall \mathbf{n} \in \mathbb{N}_{0}^{d}
$$

where $\widehat{P_{k}}(\mathbf{n}):=\sum_{\mathbf{m} \in \mathscr{L}_{k}} P_{k}(\mathbf{m}) e^{-i 2 \pi 2^{-k} \mathbf{n} \cdot \mathbf{m}}$. Related to $\phi_{k}$, the socalled $k$-scale projection operator $\mathfrak{B}_{k}$ is defined by

$$
\mathfrak{B}_{k}(f)=\sum_{\mathbf{m} \in \mathscr{L}_{k}}\left\langle f, T_{k}^{\mathbf{m}} \phi_{k}\right\rangle T_{k}^{\mathbf{m}} \phi_{k}, \quad \forall f \in H^{2}\left(\mathbb{T}^{d}\right) .
$$

Defining $\phi_{0}\left(e^{i t}\right)$ to be $\prod_{\nu=0}^{d-1}\left(1 /\left(1-\bar{a}_{\nu} e^{i t_{\nu}}\right)\right)$, the tensor product of the Cauchy kernel on the unit disc $\mathbb{D}, \mathrm{Li}$ and Qian [11] gave the expression of $\mathfrak{B}_{k}(f)$ using the analytic samples of $f$ and estimated the error $\left\|f-\mathfrak{B}_{k}(f)\right\|_{2}$ concretely.

Lemma 1 (see [11]). Let

$$
\phi_{0}\left(e^{i t}\right):=\prod_{\nu=0}^{d-1} \frac{1}{1-\bar{a}_{\nu} e^{i t_{\nu}}},
$$

where $e^{i t}=\left(e^{i t_{0}}, \ldots, e^{i t_{d-1}}\right), t=\left(t_{0}, \ldots, t_{d-1}\right) \in \mathbb{T}^{d}$, and $\left(a_{0}, \ldots, a_{d-1}\right) \in \mathbb{D}^{d}$. Construct $\phi_{k}$ by

$$
\widehat{\phi_{k}}(\mathbf{m})=\widehat{P_{k}}(\mathbf{m}) \widehat{\phi_{0}}(\mathbf{m}), \quad \forall \mathbf{m} \in \mathbb{N}_{0}^{d}, k \geq 1,
$$

where $\left\{\widehat{P_{k}}(\mathbf{m})\right\}_{\mathbf{m} \in \mathbb{N}_{0}^{d}}$ belongs to $S\left(2^{k}\right)$ such that

$$
\widehat{\phi_{k}}(\mathbf{m})=2^{-k / 2} \text {. }
$$

Then, for any $f \in H^{2}\left(\mathbb{T}^{d}\right), \mathfrak{B}_{k}(f)$ defined in (9) can be expressed by the $k$-scale analytic samples $\left\{f\left(a_{0} e^{i 2 \pi 2^{-k}\left(m_{0}^{\prime \prime}+m_{0}\right)}, \ldots, a_{d-1} e^{i 2 \pi 2^{-k}\left(m_{d-1}^{\prime \prime}+m_{d-1}\right)}\right)\right\}$ as follows:

$$
\begin{aligned}
\mathfrak{B}_{k} & (f)=\sum_{\mathbf{m} \in \mathscr{L}_{k}} \sum_{\mathbf{m}^{\prime} \in \mathscr{L}_{k}} \sum_{\mathbf{m}^{\prime \prime} \in \mathscr{L}_{k}} P_{k}\left(\mathbf{m}^{\prime}\right) \bar{P}_{k}\left(\mathbf{m}^{\prime \prime}\right) \\
\cdot & f\left(a_{0} e^{i 2 \pi 2^{-k}\left(m_{0}^{\prime \prime}+m_{0}\right)}, \ldots, a_{d-1} e^{i 2 \pi 2^{-k}\left(m_{d-1}^{\prime \prime}+m_{d-1}\right)}\right) \\
\cdot & \phi_{0}\left(e^{i\left(\cdot-2 \pi 2^{-k}\left(\mathbf{m}+\mathbf{m}^{\prime}\right)\right)}\right),
\end{aligned}
$$

where $\left\{P_{k}(\mathbf{m})\right\}_{\mathbf{m} \in \mathscr{L}_{k}}$ is the inverse Fourier transform of $\left\{\widehat{P_{k}}(\mathbf{m})\right\}_{\mathbf{m} \in \mathscr{L}_{k}}$. Moreover,

$$
\begin{aligned}
\| f- & \mathfrak{B}_{k}(f) \|_{2}^{2} \\
\leq & 2 \sum_{\mathbf{m} \in \mathscr{L}_{k}} \sum_{\mathbf{q} \in \mathbb{N}_{0}^{d} \backslash\{\mathbf{0}\}}\left|\widehat{f}\left(\mathbf{m}+2^{k} \mathbf{q}\right)\right|^{2} \\
& +\left(9\|f\|_{2}^{2}+4|\hat{f}(\mathbf{0})|^{2}\right) \frac{\sum_{\nu=0}^{d-1}\left|a_{\nu}^{2 k}\right|^{2}}{\prod_{\nu=0}^{d-1}\left(1-\left|a_{\nu}^{2^{k}}\right|^{2}\right)},
\end{aligned}
$$

where $\mathbf{0}:=(0, \ldots, 0)$.

2.2. Mathematical Materials on Multilevel Circulant Matrix. Following [12-14], we will define a multilevel circulant matrix. We begin with some denotations. For any $n \in \mathbb{N}$ and $\mathbf{n}:=\left(n_{0}, \ldots, n_{d-1}\right) \in \mathbb{N}^{d}$, let $\mathcal{N}_{n}:=\{0,1, \ldots, n-1\}$ and $\mathcal{N}_{\mathbf{n}}:=\mathcal{N}_{n_{0}} \times \cdots \times \mathcal{N}_{n_{d-1}}$, where $\times$ is the Cartesian product. An $n \times n$ matrix is referred to as a 1-level matrix of order $n$. Recursively, matrix $C_{\mathbf{n}}$ is a $d$-level matrix of order $\mathbf{n}$ if it consists of $n_{0}^{2}$ matrices of $(d-1)$-level of order $\left(n_{1}, \ldots, n_{d-1}\right)$. To point to the entries of $C_{\mathbf{n}}$, denote $C_{\mathbf{n}}$ by $\left[c_{\mathbf{i}, \mathbf{j}}\right]_{\mathbf{i}, \mathbf{j} \in \mathscr{N}_{\mathbf{n}}}$. Ad-level matrix $C_{\mathbf{n}}=\left[c_{\mathbf{i}, \mathbf{j}}\right]_{\mathbf{i}, \mathbf{j} \in \mathcal{N}_{\mathbf{n}}}$ of order $\mathbf{n}$ is referred to as a $d$-level circulant matrix if

$$
c_{\mathbf{i}, \mathbf{j}}=c_{i_{0}-j_{0}\left(\bmod n_{0}\right), \ldots, i_{d-1}-j_{d-1}\left(\bmod n_{d-1}\right)}, \quad \forall \mathbf{i}, \mathbf{j} \in \mathcal{N}_{\mathbf{n}},
$$

where $\mathbf{i}:=\left(i_{0}, \ldots, i_{d-1}\right)$ and $\mathbf{j}:=\left(j_{0}, \ldots, j_{d-1}\right)$.

An $n \times 1$ column (or $1 \times n$ row) vector is referred to as a 1 level vector of order $n$. Following the definition of a multilevel matrix, vector $V_{\mathbf{n}}$ is a $d$-level vector of order $\mathbf{n}$ if it consists of $n_{0}$ vectors of $(d-1)$-level of order $\left(n_{1}, \ldots, n_{d-1}\right)$. To point to the entries of $V_{\mathbf{n}}$, denote $V_{\mathbf{n}}$ by $\left(V_{\mathbf{n}}(\mathbf{i})\right)_{\mathbf{i} \in \mathcal{N}_{\mathbf{n}}}$.

A multilevel circulant matrix has a nice structure as shown in the following lemma.

Lemma 2 (see [12-14]). Suppose that $C_{\mathbf{n}}$ is a d-level matrix of order $\mathbf{n}$ and $\mathbf{c}_{\mathbf{n}}$ is the transpose of the first row of $C_{\mathbf{n}}$, where $\mathbf{n}=\left(n_{0}, \ldots, n_{d-1}\right)$. Then, $C_{\mathbf{n}}$ is a d-level circulant matrix if and only if

$$
C_{\mathbf{n}}=\frac{1}{\Pi_{\mathbf{n}}} \Phi_{\mathbf{n}}^{*} \operatorname{diag}\left(\Phi_{\mathbf{n}}^{*} \mathbf{c}_{\mathbf{n}}\right) \Phi_{\mathbf{n}}
$$


where $\Pi_{\mathbf{n}}:=\prod_{v=0}^{d-1} n_{v}, \Phi_{\mathbf{n}}:=F_{n_{0}} \otimes \cdots \otimes F_{n_{d-1}}$, and $\Phi_{\mathbf{n}}^{*}$ is the transpose conjugate of $\Phi_{\mathbf{n}}$. Here, $F_{n_{n}}$ is the Fourier matrix of $\operatorname{order} n_{v}$ and $\otimes$ is the Kronecker product of matrices.

Following (15), a $d$-level matrix $H_{\mathbf{n}}:=\left[h_{\mathbf{i}, \mathbf{j}}\right]_{\mathbf{i}, j \in \mathcal{N}_{\mathbf{n}}}$ of order $\mathbf{n}$ is referred to as a $d$-level Hankel matrix if

$$
h_{\mathbf{i}, \mathbf{j}}=h_{i_{0}+j_{0}, \ldots, i_{d-1}+j_{d-1}}, \quad \forall \mathbf{i}, \mathbf{j} \in \mathcal{N}_{\mathbf{n}} .
$$

Since it can be converted to a $d$-level circulant matrix by a transform to be given in Lemma 3, the Hankel matrix with the additional property

$$
h_{\mathbf{i}, \mathbf{j}}=h_{i_{0}+j_{0}\left(\bmod n_{0}\right), \ldots, i_{d-1}+j_{d-1}\left(\bmod n_{d-1}\right)}
$$

is crucial for establishing the fast algorithm in Section 2.3.

Lemma 3. Define a d-level matrix of order $\mathbf{n}$ by

$$
D_{\mathbf{n}}:=E_{n_{0}} \otimes E_{n_{1}} \otimes \cdots \otimes E_{n_{d-1}},
$$

where

$$
E_{n_{v}}:=\left[\begin{array}{ccccc}
0 & \cdots & 0 & 0 & 1 \\
0 & \cdots & 0 & 1 & 0 \\
0 & \cdots & 1 & 0 & 0 \\
\vdots & \ddots & \vdots & \vdots & \vdots \\
1 & \cdots & 0 & 0 & 0
\end{array}\right]_{n_{v} \times n_{v}}
$$

If $\mathrm{H}_{\mathbf{n}}$ is a d-level Hankel matrix of order $\mathbf{n}$ satisfying (18), then

$$
\widetilde{H}_{\mathbf{n}}:=H_{\mathbf{n}} D_{\mathbf{n}}=\left[\widetilde{h}_{\mathbf{i}, \mathbf{j}}\right]_{\mathbf{i}, \mathbf{j} \in \mathcal{N}_{\mathbf{n}}}
$$

is a d-level circulant matrix.

Proof. From (21) and (18), we arrive at

$$
\widetilde{h}_{\mathbf{i}, \mathbf{j}}=h_{\mathbf{i}, \mathbf{n}-\mathbf{1}-\mathbf{j}}=h_{i_{0}-1-j_{0}\left(\bmod n_{0}\right), \ldots, i_{d-1}-1-j_{d-1}\left(\bmod n_{d-1}\right)},
$$

where $\mathbf{1}:=(1, \ldots, 1)$. By $(22)$, if $\mathbf{i}^{\prime}:=\left(i_{0}^{\prime}, \ldots, i_{d-1}^{\prime}\right)$ and $\mathbf{j}^{\prime}:=$ $\left(j_{0}^{\prime}, \ldots, j_{d-1}^{\prime}\right)$ satisfy

$$
\begin{aligned}
i_{0}-1-j_{0}\left(\bmod n_{0}\right)= & i_{0}^{\prime}-1-j_{0}^{\prime}\left(\bmod n_{0}\right), \ldots, i_{d-1} \\
& -1-j_{d-1}\left(\bmod n_{0}\right) \\
= & i_{d-1}^{\prime}-1-j_{d-1}^{\prime}\left(\bmod n_{d-1}\right),
\end{aligned}
$$

then

$$
\widetilde{h}_{\mathbf{i}, \mathbf{j}}=h_{\mathbf{i}, \mathbf{n}-\mathbf{1}-\mathbf{j}}=h_{\mathbf{i}^{\prime}, \mathbf{n}-\mathbf{1}-\mathbf{j}^{\prime}}=\widetilde{h}_{\mathbf{i}^{\prime}, \mathbf{j}^{\prime}} .
$$

Recall that (23) is equivalent to

$$
\begin{aligned}
i_{0}-j_{0}\left(\bmod n_{0}\right)= & i_{0}^{\prime}-j_{0}^{\prime}\left(\bmod n_{0}\right), \ldots, i_{d-1} \\
& -j_{d-1}\left(\bmod n_{0}\right) \\
= & i_{d-1}^{\prime}-j_{d-1}^{\prime}\left(\bmod n_{d-1}\right),
\end{aligned}
$$

which together with (24) leads to

$$
\widetilde{h}_{\mathbf{i}, \mathrm{j}}=\widetilde{h}_{i_{0}-j_{0}\left(\bmod n_{0}\right), \ldots, i_{d-1}-j_{d-1}\left(\bmod n_{d-1}\right)} .
$$

The proof is concluded.
Note 1. It is clear that $D_{\mathbf{n}}^{-1}=D_{\mathbf{n}}$. Then, it follows from (21) that a $d$-level Hankel matrix $H_{\mathbf{n}}$ satisfying (18) can be written as

$$
H_{\mathbf{n}}=\frac{1}{\Pi_{\mathbf{n}}} \Phi_{\mathbf{n}}^{*} \operatorname{diag}\left(\Phi_{\mathbf{n}}^{*} \widetilde{\mathbf{h}}_{\mathbf{n}}\right) \Phi_{\mathbf{n}} D_{\mathbf{n}}
$$

where $\widetilde{\mathbf{h}}_{\mathbf{n}}$ is the transpose of the first row of $\widetilde{H}_{\mathbf{n}}$ given in (21).

2.3. Fast Multiscale Analytic Sampling Approximation. This subsection aims at developing a fast algorithm to compute the numerical values of $\mathfrak{B}_{k}(f)$ at $e^{i t_{\mathrm{m}, \mathbf{x}}}$, where

$$
\begin{aligned}
t_{\mathbf{m}, \mathbf{x}} & :=\mathbf{x}+2 \pi 2^{-k} \mathbf{m} \\
& =\left(x_{0}+2 \pi 2^{-k} m_{0}, \ldots, x_{d-1}+2 \pi 2^{-k} m_{d-1}\right),
\end{aligned}
$$

$\mathbf{x}:=\left(x_{0}, \ldots, x_{d-1}\right)$ is any fixed point on $\mathbb{R}^{d}$, and $\mathbf{m}=$ $\left(m_{0}, \ldots, m_{d-1}\right) \in \mathscr{L}_{k}$. For convenient narration, define a column vector $\mathbf{a}_{k, \mathbf{x}}$ by

$$
\mathbf{a}_{k, \mathbf{x}}:=\left(\mathfrak{B}_{k}(f)\left(e^{i t_{\mathbf{m}, \mathbf{x}}}\right)\right)_{\mathbf{m} \in \mathscr{L}_{k}}
$$

and a row vector $2^{\mathbf{k}}$ by $2^{\mathbf{k}}:=\left(2^{k}, \ldots, 2^{k}\right)$.

Lemma 4. Using the matrix notation, $\mathfrak{B}_{k}(f)$ in (13) can be expressed by

$$
\mathfrak{B}_{k}(f)=\left(B_{2^{\mathrm{k}}} \mathbf{P}_{2^{\mathrm{k}}}\right)^{T}\left(A_{2^{\mathrm{k}}} \overline{\mathbf{P}}_{2^{\mathrm{k}}}\right),
$$

where $A_{2^{\mathrm{k}}}$ is a matrix defined by

$$
\begin{aligned}
A_{2^{\mathrm{k}}} & :=\left[f \left(a_{0} e^{i 2 \pi 2^{-k}\left(m_{0}+m_{0}^{\prime}\right)}, \ldots,\right.\right. \\
& \left.\left.a_{d-1} e^{i 2 \pi 2^{-k}\left(m_{d-1}+m_{d-1}^{\prime}\right)}\right)\right]_{\mathbf{m}, \mathbf{m}^{\prime} \in \mathscr{L}_{k}},
\end{aligned}
$$

$\mathbf{P}_{2^{\mathbf{k}}}:=\left(P_{k, \mathbf{m}^{\prime}}\right)_{\mathbf{m}^{\prime} \in \mathscr{L}_{k}}$ is a column vector, and $B_{2^{\mathrm{k}}}$ is a matrix of functions given by

$$
B_{2^{\mathbf{k}}}:=\left[\phi_{0}\left(e^{i\left(\cdot-2 \pi 2^{-k}\left(\mathbf{m}+\mathbf{m}^{\prime}\right)\right)}\right)\right]_{\mathbf{m}, \mathbf{m}^{\prime} \in \mathscr{L}_{k}} .
$$

Moreover, $A_{2^{\mathrm{k}}}$ and $B_{2^{\mathrm{k}}}$ are both d-level Hankel matrices with property (18).

Proof. For any $\mathbf{m} \in \mathscr{L}_{k}$, it follows from (7) that

$$
\begin{aligned}
T_{k}^{\mathbf{m}} \phi_{k} & =T_{k}^{\mathbf{m}} \sum_{\mathbf{m}^{\prime} \in \mathscr{L}_{k}} P_{k, \mathbf{m}^{\prime}} T_{k}^{\mathbf{m}^{\prime}} \phi_{0}=\sum_{\mathbf{m}^{\prime} \in \mathscr{L}_{k}} P_{k, \mathbf{m}^{\prime}} T_{k}^{\mathbf{m}+\mathbf{m}^{\prime}} \phi_{0} \\
& =\sum_{\mathbf{m}^{\prime} \in \mathscr{L}_{k}} P_{k, \mathbf{m}^{\prime}} \phi_{0}\left(e^{i\left(\cdot-2 \pi 2^{-k}\left(\mathbf{m}+\mathbf{m}^{\prime}\right)\right)}\right) .
\end{aligned}
$$

Then, the column vector $\left(T_{k}^{\mathrm{m}} \phi_{k}\right)_{\mathbf{m} \in \mathscr{L}_{k}}$ can be expressed by

$$
\left(T_{k}^{\mathbf{m}} \phi_{k}\right)_{\mathbf{m} \in \mathscr{L}_{k}}=B_{2^{\mathrm{k}}} \mathbf{P}_{2^{\mathrm{k}}}
$$


By (33) and the Cauchy integral formula, for any $\mathbf{m} \in \mathscr{L}_{k}$, we have

$$
\begin{aligned}
& \left\langle f, T_{k}^{\mathbf{m}} \phi_{k}\right\rangle=\left\langle f, \sum_{\mathbf{m}^{\prime} \in \mathscr{L}_{k}} P_{k, \mathbf{m}^{\prime}} \phi_{0}\left(e^{i\left(\cdot-2 \pi 2^{-k}\left(\mathbf{m}+\mathbf{m}^{\prime}\right)\right)}\right)\right\rangle \\
& =\frac{1}{(2 \pi)^{d}} \int_{\mathbb{T}^{d}} f\left(e^{i t}\right) \\
& \sqrt{\sum_{\mathbf{m}^{\prime} \in \mathscr{L}_{k}} P_{k, \mathbf{m}^{\prime}} \phi_{0}\left(e^{i\left(t-2 \pi 2^{-k}\left(\mathbf{m}+\mathbf{m}^{\prime}\right)\right)}\right)} d t_{0} \cdots d t_{d-1} \\
& =\sum_{\mathbf{m}^{\prime} \in \mathscr{L}_{k}} \overline{P_{k, \mathbf{m}^{\prime}}} \frac{1}{(2 \pi i)^{d}} \int_{\mathbb{T}^{d}} f\left(e^{i t}\right) \\
& \cdot \prod_{\nu=0}^{d-1} \frac{1}{e^{i t_{\nu}}-a_{\nu} e^{i 2 \pi 2^{-k}\left(m_{\nu}+m_{\nu}^{\prime}\right)}} d e^{i t_{0}} \cdots d e^{i t_{d-1}} \\
& =\sum_{\mathbf{m}^{\prime} \in \mathscr{L}_{k}} \overline{P_{k, \mathbf{m}^{\prime}}} \\
& \cdot \frac{1}{(2 \pi i)^{d-1}} \int_{\mathbb{T}^{d-1}} f\left(a_{0} e^{i 2 \pi 2^{-k}\left(m_{0}+m_{0}^{\prime}\right)}, e^{i t_{1}}, \ldots, e^{i t_{d-1}}\right) \\
& \cdot \prod_{\nu=1}^{d-1} \frac{1}{e^{i t_{\nu}}-a_{\nu} e^{i 2 \pi 2^{-k}\left(m_{\nu}+m_{\nu}^{\prime}\right)}} d e^{i t_{1}} \cdots d e^{i t_{d-1}} \\
& =\sum_{\mathbf{m}^{\prime} \in \mathscr{L}_{k}} \overline{P_{k, \mathbf{m}^{\prime}}} f\left(a_{0} e^{i 2 \pi 2^{-k}\left(m_{0}+m_{0}^{\prime}\right)}, \ldots\right. \\
& \left.a_{d-1} e^{i 2 \pi 2^{-k}\left(m_{d-1}+m_{d-1}^{\prime}\right)}\right) .
\end{aligned}
$$

Therefore, the column vector $\left(\left\langle f, T_{k}^{\mathbf{m}} \phi_{k}\right\rangle\right)_{\mathbf{m} \in \mathscr{L}_{k}}$ can be rewritten as

$$
\left(\left\langle f, T_{k}^{\mathbf{m}} \phi_{k}\right\rangle\right)_{\mathbf{m} \in \mathscr{L}_{k}}=A_{2^{\mathrm{k}}} \overline{\mathbf{P}}_{2^{\mathrm{k}}}
$$

Now, the proof of (30) is concluded by (9), (34), and (36).

For any $\mathbf{m}=\left(m_{0}, m_{1}, \ldots, m_{d-1}\right)$ and $\mathbf{m}^{\prime}=$ $\left(m_{0}^{\prime}, m_{1}^{\prime}, \ldots, m_{d-1}^{\prime}\right) \in \mathscr{L}_{k}$, it is straightforward to check that

$$
\begin{gathered}
f\left(a_{0} e^{i 2 \pi 2^{-k}\left(m_{0}+m_{0}^{\prime}\right)}, \ldots, a_{d-1} e^{i 2 \pi 2^{-k}\left(m_{d-1}+m_{d-1}^{\prime}\right)}\right) \\
=f\left(a_{0} e^{i 2 \pi 2^{-k}\left(m_{0}+m_{0}^{\prime}\left(\bmod 2^{k}\right)\right)}, \ldots,\right. \\
\left.a_{d-1} e^{i 2 \pi 2^{-k}\left(m_{d-1}+m_{d-1}^{\prime}\left(\bmod 2^{k}\right)\right)}\right),
\end{gathered}
$$

and then $A_{2^{\mathrm{k}}}$ is a $d$-level Hankel matrix satisfying (18). Similarly, $B_{2^{\mathrm{k}}}$ is also a $d$-level Hankel matrix with property (18).

The following lemma is crucial for investigating the matrix expression of $\mathbf{a}_{k, \mathbf{x}}$.
Lemma 5. For any $k \in \mathbb{N}_{0}$, define a matrix of functions

$$
\mathscr{K}_{2^{k}, a}:=\left[\frac{1}{1-\bar{a} e^{i\left(\cdot-2 \pi 2^{-k}(\xi+\eta)\right)}}\right]_{\xi, \eta \in \mathcal{N}_{2^{k}}},
$$

where $a \in \mathbb{D}$. Then, for any $x \in \mathbb{R}$ and $m \in \mathcal{N}_{2^{k}}$, the value of $\mathscr{K}_{2^{k}, a}$ at $x+2 \pi 2^{-k} m$ satisfies

$$
\mathscr{K}_{2^{k}, a}\left(x+2 \pi 2^{-k} m\right)=\mathscr{K}_{2^{k}, a}(x) S_{2^{k}}^{m},
$$

where

$$
S_{2^{k}}:=\left[\begin{array}{cc}
O_{2^{k}-1,1} & I_{2^{k}-1} \\
1 & O_{1,2^{k}-1}
\end{array}\right] .
$$

Proof. We first prove by induction on $m$ that

$$
S_{2^{k}}^{m}=\left[\begin{array}{cc}
O_{2^{k}-m, m} & I_{2^{k}-m} \\
I_{m} & O_{m, 2^{k}-m}
\end{array}\right], \quad m \in \mathcal{N}_{2^{k}} .
$$

Suppose (41) holds with $m$ being replaced by $m-1$. Then,

$$
\begin{aligned}
& S_{2^{k}}^{m}=S_{2^{k}} S_{2^{k}}^{m-1} \\
& =\left[\begin{array}{cc}
O_{2^{k}-1,1} & I_{2^{k}-1} \\
1 & O_{1,2^{k}-1}
\end{array}\right]\left[\begin{array}{cc}
O_{2^{k}-m+1, m-1} & I_{2^{k}-m+1} \\
I_{m-1} & O_{m-1,2^{k}-m+1}
\end{array}\right] \\
& =\left[\begin{array}{ccc}
O_{2^{k}-1,1} & I_{2^{k}-1} \\
1 & O_{1,2^{k}-1}
\end{array}\right]\left[\begin{array}{ccc}
O_{1, m-1} & 1 & O_{1,2^{k}-m} \\
O_{2^{k}-m, m-1} & O_{2^{k}-m, 1} & I_{2^{k}-m} \\
I_{m-1} & O_{m-1,1} & O_{m-1,2^{k}-m}
\end{array}\right] \\
& =\left[\begin{array}{cc}
O_{2^{k}-m, m-1} & O_{2^{k}-m, 1} \\
I_{m-1} & O_{2^{k}-m} \\
O_{1, m-1} & O_{m-1,2^{k}-m}
\end{array}\right] \\
& =\left[\begin{array}{cc}
O_{2^{k}-m, m} & I_{2^{k}-m} \\
I_{m} & O_{m, 2^{k}-m}
\end{array}\right] .
\end{aligned}
$$

Therefore, (41) holds for any $m \in \mathcal{N}_{2^{k}}$.

For any $\xi, \eta \in \mathcal{N}_{2^{k}}$, we derive from (41) that

$$
\begin{aligned}
& {\left[\mathscr{K}_{2^{k}, a}(x) S_{2^{k}}^{m}\right]_{\xi, \eta}} \\
& = \begin{cases}{\left[\mathscr{K}_{2^{k}, a}(x)\right]_{\xi, 2^{k}-m+\eta},} & 0 \leq \eta \leq m-1 ; \\
{\left[\mathscr{K}_{2^{k}, a}(x)\right]_{\xi, \eta-m},} & m-1<\eta \leq 2^{k}-1 ;\end{cases} \\
& =\frac{1}{1-\bar{a} e^{i\left(x+2 \pi 2^{-k} m-2 \pi 2^{-k}(\xi+\eta)\right)}} \\
& =\left[\mathscr{K}_{2^{k}, a}\left(x+2 \pi 2^{-k} m\right)\right]_{\xi, \eta} .
\end{aligned}
$$

The proof of (39) is concluded.

Using (39), we will give the matrix expression of $\mathbf{a}_{k, \mathbf{x}}$ defined in (29). 
Theorem 6. Let $\left\{A_{2^{\mathrm{k}}}, B_{2^{\mathrm{k}}}, \mathbf{P}_{2^{\mathrm{k}}}\right\}$ and $S_{2^{k}}$ be as in Lemmas 4 and 5 , respectively. Define a d-level matrix $G_{2^{\mathbf{k}}}:=\left(g_{\mathbf{m}^{\prime}, \mathbf{m}}\right)_{\mathbf{m}^{\prime}, \mathbf{m} \in \mathscr{L}_{k}}$ by

$$
g_{\mathbf{m}^{\prime}, \mathbf{m}}=\left(\left(S_{2^{k}}^{m_{0}} \otimes \cdots \otimes S_{2^{k}}^{m_{d-1}}\right) \mathbf{P}_{2^{\mathbf{k}}}\right)\left(\mathbf{m}^{\prime}\right)
$$

where $\mathbf{m}=\left(m_{0}, \ldots, m_{d-1}\right)$. Then, $\mathbf{a}_{k, \mathbf{x}}$ in (29) can be expressed by

$$
\mathbf{a}_{k, \mathbf{x}}=G_{2^{\mathrm{k}}} B_{2^{\mathrm{k}}}\left(t_{0, \mathbf{x}}\right) A_{2^{\mathrm{k}}} \overline{\mathbf{P}}_{2^{\mathrm{k}}} .
$$

Proof. Using (41) to directly compute (44) gives us that

$$
\begin{aligned}
& g_{\mathbf{m}^{\prime}, \mathbf{m}}=\mathbf{P}_{2^{\mathbf{k}}}\left(m_{0}+m_{0}^{\prime}\left(\bmod 2^{k}\right), \ldots, m_{d-1}\right. \\
& \left.+m_{d-1}^{\prime}\left(\bmod 2^{k}\right)\right)
\end{aligned}
$$

from which we arrive at

$$
g_{\mathbf{m}^{\prime}, \mathbf{m}}=g_{m_{0}+m_{0}^{\prime}\left(\bmod 2^{k}\right), \ldots, m_{d-1}+m_{d-1}^{\prime}\left(\bmod 2^{k}\right)},
$$

where $\mathbf{m}^{\prime}=\left(m_{0}^{\prime}, \ldots, m_{d-1}^{\prime}\right)$. Hence, $G_{2^{\mathrm{k}}}$ is a $d$-level Hankel matrix with additional property (18).

It follows from (10), (32), (38), and (39) that the value of $B_{2^{\mathbf{k}}}$ at $t_{\mathbf{m}, \mathbf{x}}$ defined in (28) can be computed by

$$
\begin{aligned}
& B_{2^{\mathbf{k}}}\left(t_{\mathbf{m}, \mathbf{x}}\right)=\mathscr{K}_{2^{k}, a_{0}}\left(x_{0}+2 \pi 2^{-k} m_{0}\right) \otimes \cdots \\
& \otimes \mathscr{K}_{2^{k}, a_{d-1}}\left(x_{0}+2 \pi 2^{-k} m_{d-1}\right)=\left(\mathscr{K}_{2^{k}, a_{0}}\left(x_{0}\right) S_{2^{k}}^{m_{0}}\right) \\
& \quad \otimes \otimes\left(\mathscr{K}_{2^{k}, a_{d-1}}\left(x_{0}\right) S_{2^{k}}^{m_{d-1}}\right) \\
& \quad=\left(\mathscr{K}_{2^{k}, a_{0}}\left(x_{0}\right) \otimes \cdots \otimes \mathscr{K}_{2^{k}, a_{d-1}}\left(x_{0}\right)\right) \\
& \quad \cdot\left(S_{2^{k}}^{m_{0}} \otimes \cdots \otimes S_{2^{k}}^{m_{d-1}}\right)=B_{2^{\mathbf{k}}}\left(t_{\mathbf{0}, \mathbf{x}}\right) \\
& \quad \cdot\left(S_{2^{k}}^{m_{0}} \otimes \cdots \otimes S_{2^{k}}^{m_{d-1}}\right) .
\end{aligned}
$$

It is deduced from (30) and (48) that

$$
\begin{aligned}
\mathfrak{B}_{k} & (f)\left(t_{\mathbf{m}, \mathbf{x}}\right)=\left(B_{2^{\mathbf{k}}}\left(t_{\mathbf{m}, \mathbf{x}}\right) \mathbf{P}_{2^{\mathbf{k}}}\right)^{T}\left(A_{2^{\mathbf{k}}} \overline{\mathbf{P}}_{2^{\mathbf{k}}}\right) \\
= & \left(B_{2^{\mathbf{k}}}\left(t_{\mathbf{0}, \mathbf{x}}\right)\left(S_{2^{k}}^{m_{0}} \otimes \cdots \otimes S_{2^{k}}^{m_{d-1}}\right) \mathbf{P}_{2^{\mathbf{k}}}\right)^{T}\left(A_{2^{\mathbf{k}}} \overline{\mathbf{P}}_{2^{\mathbf{k}}}\right) \\
= & \left(\left(S_{2^{k}}^{m_{0}} \otimes \cdots \otimes S_{2^{k}}^{m_{d-1}}\right) \mathbf{P}_{2^{\mathbf{k}}}\right)^{T} B_{2^{\mathbf{k}}}^{T}\left(t_{\mathbf{0}, \mathbf{x}}\right)\left(A_{2^{\mathbf{k}}} \overline{\mathbf{P}}_{2^{\mathbf{k}}}\right) .
\end{aligned}
$$

By (49), the column vector $\mathbf{a}_{k, \mathbf{x}}$ defined in (29) can be expressed by

$$
\mathbf{a}_{k, \mathbf{x}}=G_{2^{\mathrm{k}}}^{T} B_{2^{\mathrm{k}}}^{T}\left(t_{\mathbf{0}, \mathbf{x}}\right) A_{2^{\mathrm{k}}} \overline{\mathbf{P}}_{2^{\mathrm{k}}} .
$$

Since both $B_{2^{\mathrm{k}}}\left(t_{0, \mathrm{x}}\right)$ and $G_{2^{\mathrm{k}}}$ are $d$-level Hankel matrices, they are both symmetric. Hence, the proof of (45) is concluded.
Note 2. Since $G_{2^{\mathrm{k}}}, B_{2^{\mathrm{k}}}\left(t_{\mathbf{0}, \mathbf{x}}\right)$, and $A_{2^{\mathrm{k}}}$ are all $d$-level Hankel matrices with property (18), by (27) and (45), $\mathbf{a}_{k, \mathbf{x}}$ can be factorized as

$$
\begin{aligned}
\mathbf{a}_{k, \mathbf{x}}= & \frac{1}{\Pi_{2^{\mathrm{k}}}} \Phi_{2^{\mathrm{k}}}^{*} \operatorname{diag}\left(\Phi_{2^{\mathrm{k}}}^{*} \widetilde{\mathbf{g}}_{2^{\mathrm{k}}}\right) \Phi_{2^{\mathrm{k}}} D_{2^{\mathrm{k}}} \frac{1}{\prod_{2^{\mathrm{k}}}} \Phi_{2^{\mathrm{k}}}^{*} \\
& \cdot \operatorname{diag}\left(\Phi_{2^{\mathrm{k}}}^{*} \widetilde{\mathbf{b}}_{2^{\mathrm{k}}}\right) \Phi_{2^{\mathrm{k}}} D_{2^{\mathrm{k}}} \frac{1}{\prod_{2^{\mathrm{k}}}} \Phi_{2^{\mathrm{k}}}^{*} \\
& \cdot \operatorname{diag}\left(\Phi_{2^{\mathrm{k}}}^{*} \widetilde{\mathbf{a}}_{2^{\mathrm{k}}}\right) \Phi_{2^{\mathrm{k}}} D_{2^{\mathrm{k}}} \overline{\mathbf{P}}_{2^{\mathrm{k}}},
\end{aligned}
$$

where $\widetilde{\mathbf{g}}_{2^{\mathrm{k}}}, \widetilde{\mathbf{b}}_{2^{\mathrm{k}}}$, and $\widetilde{\mathbf{a}}_{2^{\mathrm{k}}}$ are the transposes of the first rows of $\widetilde{G}_{2^{\mathrm{k}}}:=G_{2^{\mathrm{k}}} D_{2^{\mathrm{k}}}, \widetilde{B}_{2^{\mathrm{k}}}\left(t_{0, \mathrm{x}}\right):=B_{2^{\mathrm{k}}}\left(t_{0, \mathbf{x}}\right) D_{2^{\mathrm{k}}}$, and $\widetilde{A}_{2^{\mathrm{k}}}:=A_{2^{\mathrm{k}}} D_{2^{\mathrm{k}}}$, respectively.

Based on Note 2, a fast algorithm for $\mathbf{a}_{k, \mathbf{x}}$ will be established as follows.

Algorithm 7. Let $\phi_{0},\left\{P_{k, \mathbf{m}}\right\}_{\mathbf{m} \in \mathscr{L}_{k}}$, and $\left\{\widehat{P}_{k, \mathbf{m}}\right\}_{\mathbf{m} \in \mathscr{L}_{k}}$ be as in Lemma 1. According to (51), $\mathbf{a}_{k, \mathbf{x}}$ defined in (29) can be computed by the following six steps.

(1) By $\widehat{\phi_{0}}(\mathbf{m})=\prod_{\nu=0}^{d-1} \bar{a}_{v}^{m_{\nu}}$, (11), and (12), compute $\left\{\widehat{P}_{k, \mathbf{m}}\right\}_{\mathbf{m} \in \mathscr{L}_{k}}$, where $\mathbf{m}=\left(m_{0}, \ldots, m_{d-1}\right)$.

(2) By implementing IFFT on $\left\{\widehat{P}_{k, \mathbf{m}}\right\}_{\mathbf{m} \in \mathscr{L}_{k}}$, we compute $\mathbf{P}_{2^{\mathrm{k}}}$.

(3) Since $\Phi_{2^{\mathrm{k}}}=F_{2^{k}} \otimes \cdots \otimes F_{2^{k}}$ and $F_{2^{k}}$ is the Fourier matrix of order $2^{k}, \Phi_{2^{\mathrm{k}}}^{*} \widetilde{\mathbf{g}}_{2^{\mathrm{k}}}, \Phi_{2^{\mathrm{k}}}^{*} \widetilde{\mathbf{a}}_{2^{\mathrm{k}}}$, and $\Phi_{2^{\mathrm{k}}}^{*} \widetilde{\mathbf{b}}_{2^{\mathrm{k}}}$ are computed by IFFTs.

(4) Consider

$$
\begin{aligned}
\mathbf{P}_{2^{\mathrm{k}}} \stackrel{D_{2^{\mathrm{k}}}}{\longrightarrow} D_{2^{\mathrm{k}}} \overline{\mathbf{P}}_{2^{\mathrm{k}}} \stackrel{\mathrm{FFT}}{\longrightarrow} \Phi_{2^{\mathrm{k}}} D_{2^{\mathrm{k}}} \overline{\mathbf{P}}_{2^{\mathrm{k}}} \\
\stackrel{\operatorname{diag}\left(\Phi_{2^{\mathrm{k}}}^{*} \widetilde{\mathbf{a}}_{2^{\mathrm{k}}}\right)}{\longrightarrow} \operatorname{diag}\left(\Phi_{2^{\mathrm{k}}}^{*} \widetilde{\mathbf{a}}_{2^{\mathrm{k}}}\right) \Phi_{2^{\mathrm{k}}} D_{2^{\mathrm{k}}} \overline{\mathbf{P}}_{2^{\mathrm{k}}} \stackrel{\text { IFFT }}{\longrightarrow} \mathbf{a}_{k, \mathbf{x}}^{1} \\
:=\frac{1}{\prod_{2^{\mathrm{k}}}} \Phi_{2^{\mathrm{k}}}^{*} \operatorname{diag}\left(\Phi_{2^{\mathrm{k}}}^{*} \widetilde{\mathbf{a}}_{2^{\mathrm{k}}}\right) \Phi_{2^{\mathrm{k}}} D_{2^{\mathrm{k}}} \overline{\mathbf{P}}_{2^{\mathrm{k}}} .
\end{aligned}
$$

(5) Consider

$$
\begin{aligned}
\mathbf{a}_{k, \mathbf{x}}^{1} \stackrel{D_{2^{\mathrm{k}}}}{\longrightarrow} D_{2^{\mathrm{k}}} \mathbf{a}_{k, \mathbf{x}}^{1} \stackrel{\mathrm{FFT}}{\longrightarrow} \Phi_{2^{\mathrm{k}}} D_{2^{\mathrm{k}}} \mathbf{a}_{k, \mathbf{x}}^{1} \\
\stackrel{\operatorname{diag}\left(\Phi_{2^{\mathbf{k}}}^{*} \widetilde{\mathbf{b}}_{2^{\mathrm{k}}}\right)}{\longrightarrow} \operatorname{diag}\left(\Phi_{2^{\mathrm{k}}}^{*} \widetilde{\mathbf{b}}_{2^{\mathrm{k}}}\right) \Phi_{2^{\mathrm{k}}} D_{2^{\mathrm{k}}} \mathbf{a}_{k, \mathbf{x}}^{1} \stackrel{\text { IFFT }}{\longrightarrow} \mathbf{a}_{k, \mathbf{x}}^{2} \\
:=\frac{1}{\Pi_{2^{\mathrm{k}}}} \Phi_{2^{\mathrm{k}}}^{*} \operatorname{diag}\left(\Phi_{2^{\mathrm{k}}}^{*} \widetilde{\mathbf{b}}_{2^{\mathrm{k}}}\right) \Phi_{2^{\mathrm{k}}} D_{2^{\mathrm{k}}} \mathbf{a}_{k, \mathbf{x}}^{1} \cdot
\end{aligned}
$$

(6) Consider

$$
\begin{aligned}
\mathbf{a}_{k, \mathbf{x}}^{2} & \stackrel{D_{2^{\mathrm{k}}}}{\longrightarrow} D_{2^{\mathrm{k}}} \mathbf{a}_{k, \mathbf{x}}^{2} \stackrel{\text { FFT }}{\longrightarrow} \Phi_{2^{\mathrm{k}}} D_{2^{\mathrm{k}}} \mathbf{a}_{k, \mathbf{x}}^{2} \\
& \stackrel{\operatorname{diag}\left(\Phi_{2^{\mathrm{k}}}^{*} \widetilde{\mathbf{b}}_{2^{\mathrm{k}}}\right)}{\longrightarrow} \operatorname{diag}\left(\Phi_{2^{\mathrm{k}}}^{*} \widetilde{\mathbf{g}}_{2^{\mathrm{k}}}\right) \Phi_{2^{\mathrm{k}}} D_{2^{\mathrm{k}}} \mathbf{a}_{k, \mathbf{x}}^{2} \stackrel{\text { IFFT }}{\longrightarrow} \mathbf{a}_{k, \mathbf{x}} \\
= & \frac{1}{\Pi_{2^{\mathrm{k}}}} \Phi_{2^{\mathrm{k}}}^{*} \operatorname{diag}\left(\Phi_{2^{\mathrm{k}}}^{*} \widetilde{\mathbf{g}}_{2^{\mathrm{k}}}\right) \Phi_{2^{\mathrm{k}}} D_{2^{\mathrm{k}}} \mathbf{a}_{k, \mathbf{x}}^{2} .
\end{aligned}
$$


TABLE 1: The error ratios (i.e., ratio 1, ratio 2, and ratio 3) corresponding to different $k, a_{0}$, and $a_{1} . T_{1}$ and $T_{2}$ are the time cost by direct computing and Algorithm 7, respectively.

\begin{tabular}{cccccccc}
\hline$k$ & $a_{0}$ & $a_{1}$ & ratio 1 & ratio 2 & ratio 3 & $T_{1}$ \\
\hline 2 & 0.01 & 0.32 & 0.0704 & 0.0733 & 0.0676 & $0.107 \mathrm{~s}$ & $0.086 \mathrm{~s}$ \\
3 & 0.08 & 0.16 & 0.0051 & 0.0051 & 0.0051 & $0.214 \mathrm{~s}$ & $0.103 \mathrm{~s}$ \\
4 & 0.44 & 0.41 & $2.5618 \times 10^{-5}$ & $2.5652 \times 10^{-5}$ & $2.5584 \times 10^{-5}$ & $4.052 \mathrm{~s}$ & $0.116 \mathrm{~s}$ \\
\hline
\end{tabular}

Computational Complexity. It is easy to check that directly computing $\mathbf{a}_{k, \mathbf{x}}$ through (13) costs $\mathcal{O}\left(2^{3 d k}\right)$ operations. In Algorithm 7, however, FFT and IFFT are used for three and seven times, respectively, which cost $\mathcal{O}\left(2^{k d} \cdot d k\right)$ operations. Meanwhile, the complexity of other operations such as the multiplication of $D_{2^{\mathrm{k}}}$ and $\overline{\mathbf{P}}_{2^{\mathrm{k}}}$ is $\mathcal{O}\left(2^{k d}\right)$. Therefore, the computational complexity of Algorithm 7 is $\mathscr{O}\left(2^{k d} \cdot d k\right)$.

Note 3. So far, on computing $\mathbf{a}_{k, \mathbf{x}}$, we have not found any numerical algorithm better than Algorithm 7. On the other hand, we notice that Algorithm 7 holds for equally spaced points on $\mathbb{T}^{d}$. In another occasion, we will establish the fast algorithm for the case of nonequally spaced points.

\section{Experiment}

To check the efficiency of Algorithm 7, a numerical computation experiment on the function

$$
f\left(e^{i t}\right)=\frac{e^{i t_{1}}\left(1+0.3 e^{i t_{0}}\right)}{1.5+0.4 e^{i\left(t_{0}+2 t_{1}\right)}}, \quad t=\left(t_{0}, t_{1}\right) \in \mathbb{T}^{2},
$$

will be carried out. Table 1 shows the approximation error ratios

$$
\begin{aligned}
& \text { ratio } 1:=\frac{\left\|\mathbf{b}_{k, \mathbf{0}}-\mathbf{a}_{k, \mathbf{0}}\right\|_{\ell_{2}}}{\left\|\mathbf{b}_{k, \mathbf{0}}\right\|_{\ell_{2}}}, \\
& \text { ratio } 2:=\frac{\left\|\operatorname{Re}\left(\mathbf{b}_{k, \mathbf{0}}-\mathbf{a}_{k, \mathbf{0}}\right)\right\|_{\ell_{2}}}{\left\|\operatorname{Re}\left(\mathbf{b}_{k, \mathbf{0}}\right)\right\|_{\ell_{2}}}, \\
& \text { ratio } 3:=\frac{\left\|\operatorname{Im}\left(\mathbf{b}_{k, \mathbf{0}}-\mathbf{a}_{k, \mathbf{0}}\right)\right\|_{\ell_{2}}}{\left\|\operatorname{Im}\left(\mathbf{b}_{k, \mathbf{0}}\right)\right\|_{\ell_{2}}},
\end{aligned}
$$

and the times $T_{1}$ and $T_{2}$ corresponding to different choices of $k, a_{0}$, and $a_{1}$, where $\mathbf{b}_{k, 0}=\left(f\left(e^{i t_{\mathbf{m}, 0}}\right)\right)_{\mathbf{m} \in \mathscr{L}_{k}}$ and $T_{1}$ and $T_{2}$ are the compute running times cost by directly computing $\mathbf{a}_{k, 0}$ and Algorithm 7, respectively. The data on the time cost confirms that Algorithm 7 is faster than direct computing. Therefore, the result in this experiment matches the computational complexity analysis in Section 2. On the other hand, as for the approximation accuracy, as the scale level $k$ increases, the approximation efficiency becomes better. This coincides with the approximation error estimation given in (14).

\section{Competing Interests}

The authors declare that they have no competing interests.

\section{Acknowledgments}

The work is supported by National Natural Science Foundation of China (Grant nos. 61561006, 11501132, and 11461002), Guangxi Natural Science Foundation (Grant no. 2013GXNSFBA019010), and Natural Science Foundation of Guangdong Province (Grant no. 2015A030313443).

\section{References}

[1] W. Sun and X. Zhou, "Reconstruction of band-limited functions from local averages," Constructive Approximation, vol. 18, no. 2, pp. 205-222, 2002.

[2] G. G. Walter, "A sampling theorem for wavelet subspaces," IEEE Transactions on Information Theory, vol. 38, no. 2, pp. 881-884, 1992.

[3] G.-C. Wu, Z.-X. Cheng, and X.-H. Yang, “The cardinal orthogonal scaling function and sampling theorem in the wavelet subspaces," Applied Mathematics and Computation, vol. 194, no. 1, pp. 199-214, 2007.

[4] G. Wu, D. Li, and H. Cao, "Necessary and sufficient conditions of the wave packet frames in $L_{2}\left(R_{n}\right)$," Bulletin of the Malaysian Mathematical Sciences Society. Second Series, vol. 37, no. 4, pp. 1123-1136, 2014.

[5] G. Wu and D. Li, "Characterizations of the multivariate wave packet systems," Taiwanese Journal of Mathematics, vol. 18, no. 5, pp. 1389-1409, 2014.

[6] Y. Fu and L. Li, "Nontrivial harmonic waves with positive instantaneous frequency," Nonlinear Analysis: Theory, Methods \& Applications, vol. 68, no. 8, pp. 2431-2444, 2008.

[7] T. Qian, Q. Chen, and L. Li, "Analytic unit quadrature signals with nonlinear phase," Physica D: Nonlinear Phenomena, vol. 203, no. 1-2, pp. 80-87, 2005.

[8] T. Qian, "Mono-components for decomposition of signals," Mathematical Methods in the Applied Sciences, vol. 29, no. 10, pp. 1187-1198, 2006.

[9] T. Qian and Y.-B. Wang, "Adaptive Fourier seriesła variation of greedy algorithm," Advances in Computational Mathematics, vol. 34, no. 3, pp. 279-293, 2011.

[10] Q. Chen, W. Mai, L. Zhang, and W. Mi, "System identification by discrete rational atoms," Automatica, vol. 56, pp. 53-59, 2015.

[11] Y. Li and T. Qian, "Analytic sampling approximation by projection operator with application in decomposition of instantaneous frequency," International Journal of Wavelets, Multiresolution and Information Processing, vol. 11, no. 5, Article ID 1350040, 2013.

[12] P. J. Davis, Circulant Matrices, John Wiley \& Sons, New York, NY, USA, 1979. 
[13] E. E. Tyrtyshnikov, "A unifying approach to some old and new theorems on distribution and clustering," Linear Algebra and Its Applications, vol. 232, pp. 1-43, 1996.

[14] S. Serra Capizzano and E. Tyrtyshnikov, "Any circulant-like preconditioner for multilevel matrices is not superlinear," SIAM Journal on Matrix Analysis \& Applications, vol. 21, no. 2, pp. 431439, 2000. 


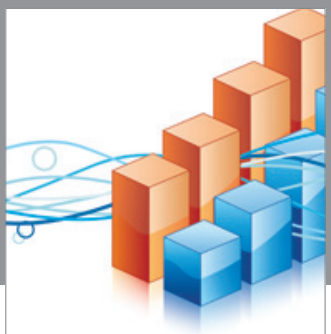

Advances in

Operations Research

vatem alat4

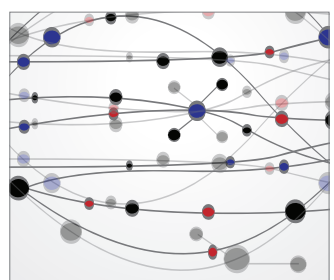

\section{The Scientific} World Journal
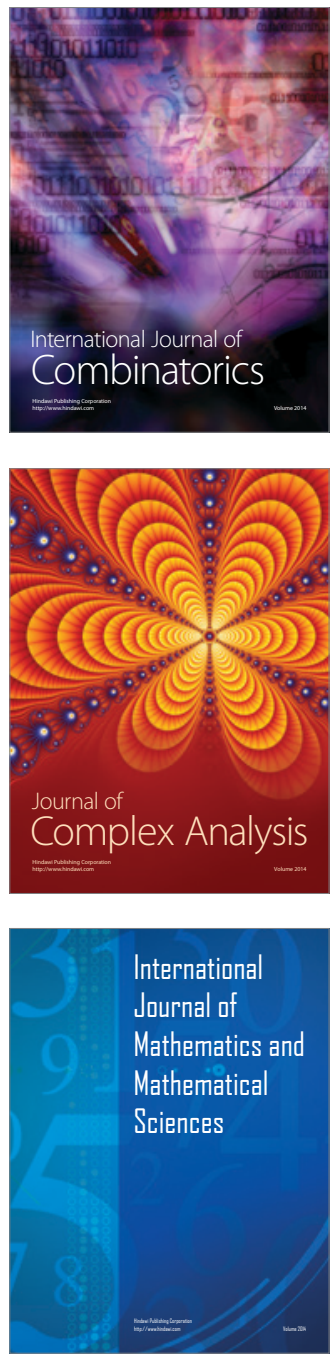
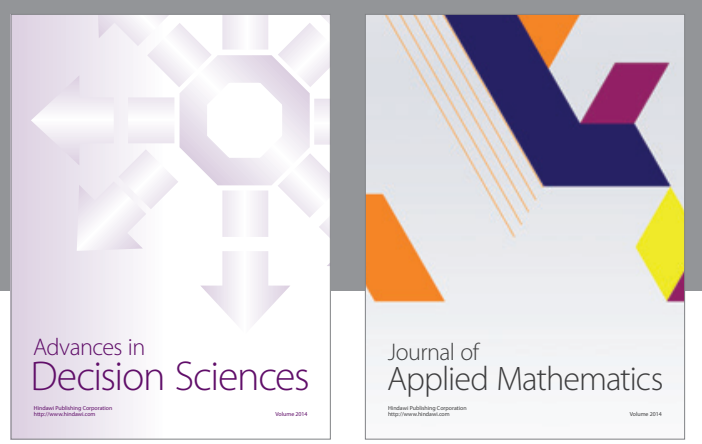

Algebra

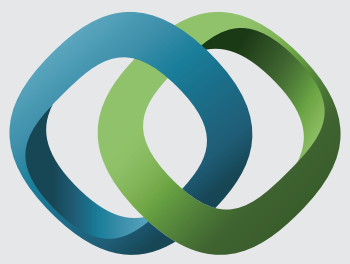

\section{Hindawi}

Submit your manuscripts at

http://www.hindawi.com
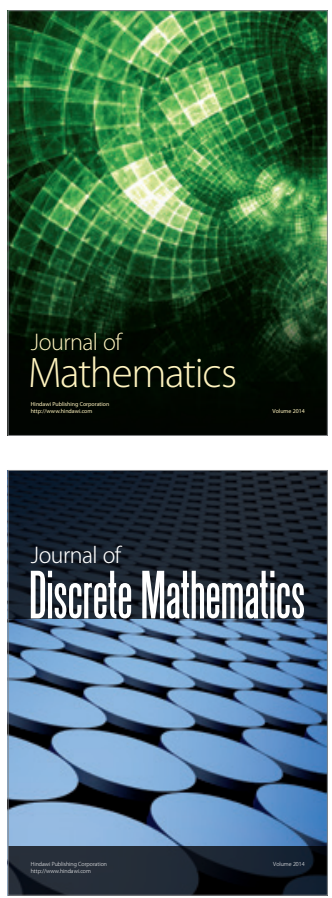

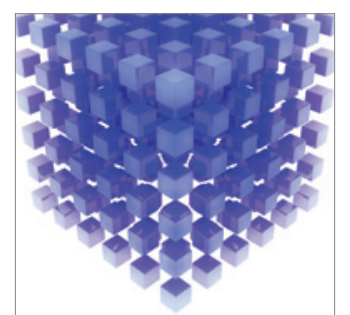

Mathematical Problems in Engineering
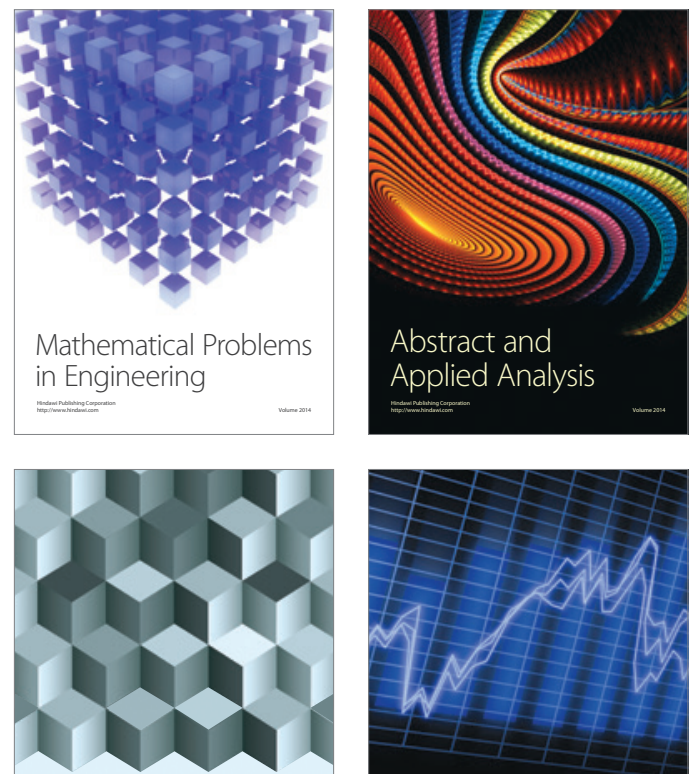

Journal of

Function Spaces

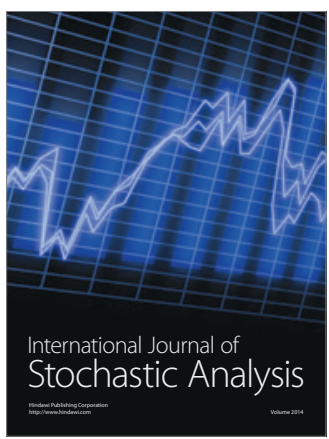

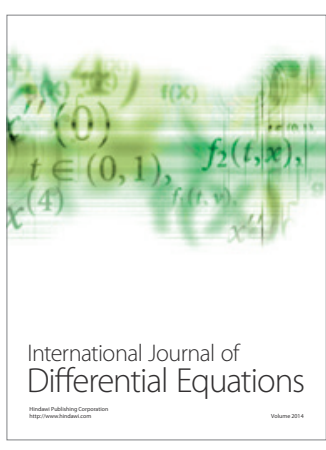
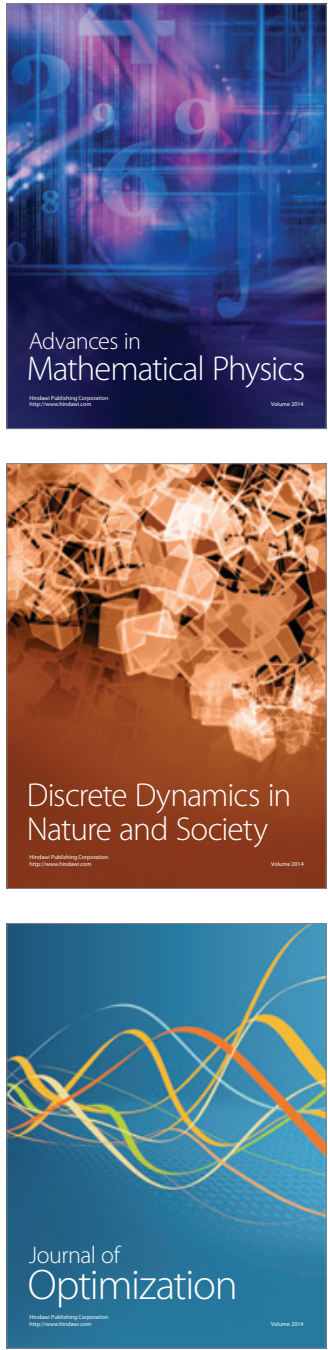